\title{
The Importance of Thinking Coherently in Strategic Asset Allocation
}

\author{
Philippe J.S. De Brouwer ${ }^{\#, *}$
}

University of Warsaw, Poland

\begin{abstract}
This paper is part one of an homage to the seminal paper of Artzner, Delbaen, Eber, and Heath (1997) [1], who proposed a set of axioms that must be satisfied by risk measures in order to be "coherent".

This paper does not aim to add to the knowledge of coherent risk measures, but it aims to prove that coherence matters not only for the mathematician, but also for the investment manager and his clients by constructing simple and transparent examples that show the dangers of working with incoherent risk measures. This way the author hopes to improve communication between the academic communities on one side and on the others side policy-makers and operational decision makers at financial institutions, their regulators and law-makers, who fifteen year after that paper still underestimate the importance of "thinking coherently".
\end{abstract}

Keywords: Portfolio Selection, Personal Financial Decision Making, Coherent Risk Measures, Strategic Asset Allocation, Suitability of Investments, Value at Risk, Expected Shortfall, Global Exposure, Risk Classification, risk and reward indicator, variance, UCITS IV, FINRA 2011.

\section{INTRODUCTION}

Bernoulli (1738) [2] recognized that the risk averse investor should seek to diversify, Fisher (1906) [3] proposed variance as a measure for economic risk, and Marschak (1938) ${ }^{1}$ [4] suggested to use mean and variance as parameters in utility (for consumption of commodities). However we had to wait till Markowitz (1952a) [5] for the first explicit quantification and identification of investment risk, when he proposed his mean-variance criterion. After almost 60 years his method is often used by practitioners and remains a reference for scholars (e.g. Rubinstein, 2002 [6]). Markowitz used in his seminal paper of 1952 variance as risk measure. He did not discus in that paper the choice for variance but argued that the mean-variance criterion was better than simply chasing return because it stimulated diversification. This makes Markowitz the first to argue that the acid test for a portfolio optimization method was that it leads to diversification.

*Correspondence Address to this author at the University of Warsaw, Poland; E-mail: philippe@de-brouwer.com

JEL Classification Numbers: C58, G11, G18, G28, G32.

\footnotetext{
\#Philippe J.S. De Brouwer holds a PhD in finance and a M.Sc. degrees in Theoretical Physics and Applied Economic Sciences (Management Science). Active for over 20 years in Investment Management, working in Belgium, Poland, UK and Ireland at Deutsche Bank, Fortis, and KBC, and RBS he oversaw the birth of structured funds in Belgium and Poland, presided over a merger of asset managers, and subsequently In creased market share as CEO of KBC TFI in Poland. He over Saw more than 24 bln EUR in about a thousand portfolios as director and member of the board at KBC Fund Management Ltd. and currently he is Director at HSBC Bank, professor at the University of Warsaw as well as lecturer at Vlerick Business School.

${ }^{1}$ Marschak supervised Markowitz dissertation, but we were unable to find a reference in Markowitz' papers to Marschalk's paper. Markowitz relates in his 1991 Noble lecture Markowitz (1991) [8] that the idea came to him while reading Williams (1938) [44].
}

Seven years later in his book "Portfolio Selection, Efficient Diversification of Investments" (Markowitz, 1959 [7]) he suggested semi-variance and in his Noble Lecture on 7 December 1990 (published in Markowitz (1991) [8]) he defined semi-variance relative to an investment target, $c$.

$$
S:=E\left[\min (0, R-c)^{2}\right]
$$

Hereby reconnecting to his line of thinking from Customary Wealth Theory (see Markowitz, 1952b [9]) and the seminal paper of Roy (1952) [10] -that identifies the Safety First Theory-where the investment goal is key. Doing so, Markowitz further laid the foundations of a different approach, alternative to his own mean-variance approach, that defines risk relative to one or more investment targets-as opposed to considering all assets in one portfolio as in the mean-variance approach. This line of thinking paved the way for many different developments such as Maslowian Portfolio Theory (see De Brouwer, 2009 [11]), Target Oriented Investment Advice (see De Brouwer, 2011 [12]), the asset-liability approach (see Amenc, Martellini, Milhau, and Ziemann, 2009 [13]). To some extend Markowitz' and Roy's 1952 [10] theories can be considered as the foundation of Behavioural Portfolio Theory (see Shefrin and Statman, 2000 [14]). ${ }^{2}$ In this approach risk cannot be defined without the investment target being specified, risk is inherently connected to not attaining the investment goal. This means for example that a zero return is not necessarily

\footnotetext{
${ }^{2}$ Actually, the Single Account version of Behavioural Finance is a direct descendent of Roy's Safety First theory, via Lopez (1987)'s [32] SP/A theory and Tesler (1955)'s [38] generalization of the Safety First Theory. The major contribution of the Behavioural Portfolio Theory is the formulation of the Multiple Account version, allowing for multiple invest- ment goals.
} 
risk-less because it might under-perform investment target, which is most probably inflation linked.

Markowitz' already wrote that "semi-variance looks more plausible than variance" and that he "proposed semi-variance" (see Markowitz, 1991 [8]). In his 1959 book he provides on pg. 193 and 194 some arguments why in practice variance is a good choice. He argues that he did not find an important class of utility functions that would be coherent with semi-variance and not with variance, and that therefore-taken into account that variance is more familiar and faster to calculatevariance could be something like a zero hypothesis.

Actually it is not so difficult to find a class of utility functions that is coherent with semi-variance and not with variance. Each investor, who is loss averse, will find that his or her utility function will not have variance as a parameter, but that semi-variance can be a reasonable parameter in his or her utility function. Especially when asset returns do not follow a Gaussian distribution, variance looses quickly its appeal as we will see in this paper.

\section{THINKING COHERENTLY}

\section{Definition of Coherent Risk Measures}

Considering the question of how financial risk could be described, Artzner, Delbaen, Eber, and Heath (1997) [1] proposed the following set of axioms ${ }^{3}$ :

\section{Axiom (Coherent Risk Measure)}

A function $\rho: \mathbb{V} \mapsto \mathbb{R}$ (where $\mathbb{V}$ is the set of real-valued stochastic variables) is called a coherent risk measure if and only if it is

\section{1. monotonous:}

$\forall X, Y \in \mathbb{V}: X \leq Y \Rightarrow \rho(X) \geq \rho(Y)$

If a portfolio $Y$ has always better results than portfolio $X$, then its risk is less.

2. sub-additive:

$\forall X, Y, X+Y \in \mathbb{V}:$

$\rho(X+Y) \leq \rho(X)+\rho(Y)$

Diversification reduces risk: the risk of a portfolio cannot be worse than that of any of its constituents separately.

${ }^{3}$ For a profound elaboration, please refer to Artzner, Delbaen, Eber, and Heath (1999) [24].
3. positively homogeneous:

$\forall a>0$ and $\forall X, a X \in \mathbb{V}$ :

$\rho(a X)=a \rho(X)$

Investing a times more money increases the risk a times.

\section{4. translational invariant:}

$\forall c>0$ and $\forall X \in \mathbb{V}:$

$\rho(X+c)=\rho(X)-c$

Adding an amount $c$ in cash to the portfolio reduces the risk with $c$.

These axioms seem to be congruent with what one intuitively understands as "risk" in a financial context. Axiom two states for example that diversification reduces risk. Indeed when one owns two assets, the outcome is in the worst case the result of the two worst case scenarios. In all other cases the outcome of the diversified portfolio is better. This is so fundamental in our thinking about financial risk that Artzner, Delbaen, Eber and Heath [1] elected this as an axiom for coherence and that Markowitz (1952a) [5] considered this aspect as the key test for his new mean variance criterion. Some authors, such as Acerbi and Tasche (2002a) [15] consider only risk measures that are coherent in this sense and all other measures, such as Value at Risk ( $V a R$ henceforth), variance (VAR henceforth) and standard deviation, are not considered as a risk measure at all.

An important footnote is that these axioms are only realistic when markets are liquid and when the investor would never be in a position to influence the price. This phenomenon is pretty much the same as the Euclidean axioms of geometry, that generally work fine, but fail to describe reality in the presence of large masseswhere one needs the theory of General Relativity (see Einstein, 1916 [16]). Also, similar to the Euclidean axioms, this does not mean that this is the only set of possible axioms. For example it is possible to develop internally coherent geometries with different axioms, such as Lobachevsky and Bolyai's geometry on the surface of a pseudosphere, or the geometry on a sphere, developed by Riemann. While none of these sets of axioms can be wrong or right, only the Euclidean geometry makes sense for applications such as building a house or a rail-road. In the same way we will illustrate that Artzner, Delbaen, Eber, and Heath's [1] set of axioms lead to a coherent representation of risk in liquid financial markets for investors who cannot influence price. 


\section{The Consequences of Thinking Incoherently}

Let's illustrate the consequences of failing to select a coherent risk measure with a simple example. First we define Value at Risk ( $\operatorname{VaR})$.

\section{Definition (Value at Risk (VaR))}

For the real-valued stochastic profit variable, return $(X)$, and a probability alpha, $\alpha \in[0,1]$, the Value at Risk (VaR) is defined as

$\operatorname{VaR}_{\alpha}(X):=-q_{\alpha}(X)$

with $q_{\alpha}(X)$ the $\alpha$-quantile of the stochastic variable $X$.

Often this is worded as "the $V a R$, given a confidence interval of $(1-\alpha)$, is that value so that there is a probability of $\alpha$ to have an outcome worse than $V a R$." In other words, the $\alpha-V a R$ is the best outcome of the $100 \alpha \%$ worst outcomes.

Let us start with an example, similar to the one used in Acerbi (2004) [17]. ${ }^{4}$

Example 2.1. Assume one bond that pays in one year a coupon of $5 \%$ plus the captial back with a probability of $99.3 \%$. So, there is a $0.7 \%$ probability to default.

Its $1 \% \mathrm{VaR}$ is $-5 \%$, because the only default cases are below the $1 \%$ quantile. In other words, VaR spots no risk!

Example 2.2. Now consider two identical bonds with the same parameters, but independently distributed (say, one bond on an Indian ICT company and another on a Canadian diamond mine).

Clearly a portfolio that holds $50 \%$ of each must be less risky. Only in the extreme improbable case (probability $=0.000049$ ) that both default we lose all our money, in all other cases at least $50 \%$ of the portfolio is preserved. Diversification reduces the risk.

However calculating the $1 \% \mathrm{VaR}$ of the diversified portfolio one finds that it equals $47.5 \%$ of the portfolio!

Example 2.3. Consider now $N$ such identically and independently distributed bonds.

$V a R$ and $E S$ behave then -in function of $N-$ as presented in Figure 1.

\footnotetext{
${ }^{4}$ For the reader it might be comforting to know that the results are exactly the same, even while our method of calculating was different. We used a numerical approach by convolving the underlying distributions and Acerbi (2004) [17] used binomial distributions.
}

This is a dramatic example of what can happen when one of the axioms is not respected. $V a R$ is not sub-additive, and hence can and will lead to absurd results. Using VaR to measure risk is similar to using a rubber band in order to measure length. Because of the lack of sub-additivity $\operatorname{VaR}$ can counteract diversification. With hindsight, this seems to be a parsimonious factor in the fate of the investment bank Lehman Brothers: on the assets side of their balance they increasingly relied on a less diversified real estate holding, while reporting $V a R$ numbers to the authorities. ${ }^{5}$

It has to be said, however, that when distributions are "nicely bell-shaped"6, VaR will give a good indication of risk.

In Figure 1 we present an overview of what happens to the $V a R$ when the portfolio is more and more diversified. There is not only a false minimum at one bond, but also at 21 and 63 ! These false minima are not only counter-intuitive, but make $V a R$ useless for portfolio optimization. When the risk surface is not concave, then it is not possible to make an optimization and trust the results.

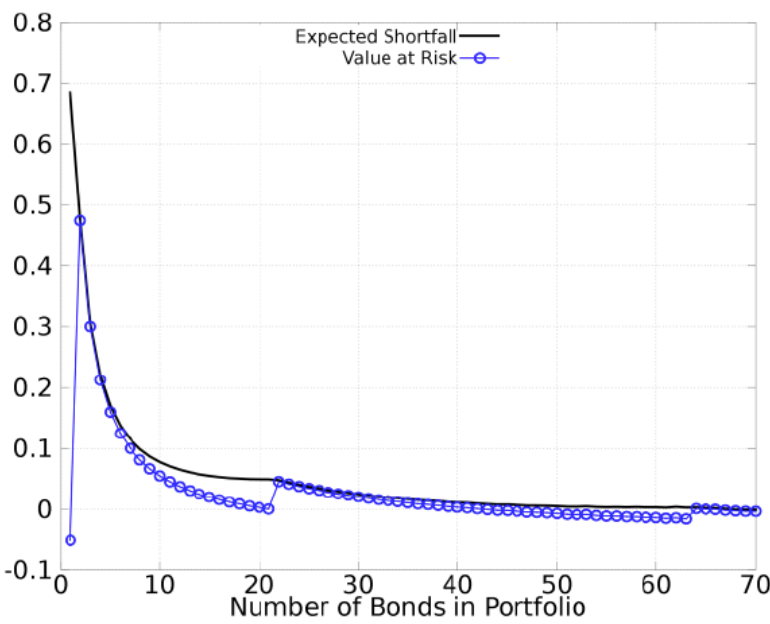

Figure 1: The risk associated to Example 2.3. On the $\mathrm{x}$-axis we present an increasing number of independent bonds in the portfolio. On the y-axis the fat line without dots is $E S_{0.01}$, the one percent expected shortfall-see Definition 2.2, and the narrow line with dots is the $\operatorname{VaR}_{0.01}$. Note that $\operatorname{VaR}$ displays a false minimum for 1,21 and 63 bonds.

${ }^{5}$ Some insight in the balance sheet of Lehman Brothers can be found in McDonald and Robinson (2009) [33], or in its annual statements of accounts

${ }^{6} \mathrm{~A}$ mathematical correct way of expressing this "nice bell-shape" is via elliptical distribution functions. $\mathrm{VaR}$ is sub-additive for elliptically distributed asset returns. An elliptical distribution function is defined as a distribution for which the characteristic function only depends on one variable: $x^{\prime} \sum x$, where $\sum$ is a positive definite matrix. A characteristic of these distribution functions is that in the two-dimensional case the iso-density curves form ellipses (and in higher dimensions ellipsoids), however this property is not a suficient condition to be an elliptical function. The multivariate normal, logistic, exponential power, Cauchy, Laplace and $t$-distributions for example belong to the class of elliptical distributions. 
One might argue that the viewpoint is important. Indeed, let us rethink the previous examples, but not from the investor's point of view: from the banker's point of view. Further assume that this banker has a no guilt feelings, and that this banker will loose the client if a loss occurs. In this case the banker could then decide to minimize the probability that a loss occurs and just pick one bond. In total disrespect for the investor, the minimal-VaR portfolio can-from this point of view-be an optimal choice. But note that this does not solve the issues of convexity. The absence of convexity is an indication that axioms allowing $V a R$ would not be internally coherent.

The axioms under which VaR would be coherent still have to be written. It seems to us that monotonicity, positively homogeneity together with a third axiom that states that all outcomes below a certain probability level are lethal (so that the amplitude of loss is irrelevant) could be a possible set of axioms under which $V a R$ is coherent. A person with a sound mind would however not find that these axioms describe his or her thinking about financial risk in a sufficient accurate way.

\section{Is There a Coherent Alternative?}

Surprisingly, practitioners, law makers and regulators continued to embrace $V a R$ and $V a R$ accompanied us to the 2008 Global Meltdown. Even later implementation measures of UCITS IV enforce the use of $V a R$, even at the level of individual investment funds. ${ }^{7}$

Quite probably $\operatorname{VaR}$ is so popular for its simplicity, ease of calculation, universality, and seemingly (but deceivingly) ease of interpretation. $V a R$ is the answer to the simple question: "What is the lowest amount that one can expect to loose in the $100 \alpha \%$ worst cases?". On top of that, it is expressed in the same units as the portfolio, and is straightforward to calculate. In the years that followed Artzner, Delbaen, Eber, and Heath (1997)'s [1] publication there was no coherent alternative for $V a R$ known that combined all of the advantages that made it so popular.

Pflug (2000) [18] proved that the answer to the following simple question: "What is the average amount of loss in the $100 \alpha \%$ worst cases?" leads to a coherent risk measure. This risk measure goes by many names: Conditional Value at Risk ( $C V a R)$, Expected Shortfall (ES), Average Value at Risk ( $A V a R)$, Expected Tail Risk (ETR), and for continuous distributions it coincides with the Tail Conditional Expectation (TCE), which is not necessarily sub-

\footnotetext{
${ }^{7}$ An exception if for example Yamai and Yoshiba (2002b) [46], where officials of the Bank of Japan embrace the concept of coherence.
}

additive for non-continuous distributions. We will choose for the name Expected Shortfall (ES henceforth), as it seems the most clarifying and pure. We define $E S$ as follows.

\section{Definition (Expected Shortfall (ES))}

For a real-valued stochastic profit variable, $X$ (such as return), the Expected Shortfall is the expected loss in the $100 \alpha \%$ worst observations

$E S_{(\alpha) N}(X):=-\frac{\sum_{i=1}^{n} X_{i \cdot N}}{n}$

$=-($ average of the worst $100 \alpha \%$ realizations $)$

Where the index $i: N$ refers to the index of the observations, after sorting from the worst to the best observations, and $n$ is a number to be determined in function of $\alpha$.

Acerbi and Tasche (2002b) [19] show that this is equivalent with;

\section{Definition (Expected Shortfall)}

$$
E S_{(\alpha)}(X):=-\frac{1}{\alpha} \int_{0}^{\alpha} F^{\leftarrow}(p) d p
$$

with $\quad F^{\leftarrow}(p):=\inf \{x \mid F(x) \geq p\} \quad$ the leftgeneralized inverse of the cumulative distribution function of $X$. For continuous distributions one has $F^{\leftarrow}(p)=q_{\alpha}(X)$.

This definition makes immediately clear that $E S$ is continuous in $\alpha$. This is a property that has conceptual importance, and is not possessed by $V a R$, for example. Figure 2 show how counter-intuitive this makes $V a R$.

Corollary 2.1. For continuous distributions, this definition reduces to

$$
E S_{(\alpha)}(X)=-\frac{1}{\alpha} \int_{0}^{\alpha} Q_{X}(p) d p
$$

with $Q(p)$ the quantile function (or the inverse of the cumulative distribution function).

Which is then also equivalent to

$$
E S_{(\alpha)}(X)=-\frac{1}{\alpha} \int_{0}^{q_{\alpha}(X)} x f_{x}(x) d x
$$

So, since 2000 we have a coherent alternative for $V a R$ that has all its advantages: simple interpretation, expressed in monetary unit of measurement and universality of use. However there is a drawback: ES 
might be more difficult to estimate. Indeed, where $V a R$ simply ignores tail of the distribution, ES will take all worst outcomes into account. This makes ES more sensitive to estimation errors in the tails of the distributions. However we note that there are many ways to simplify the calculations. ${ }^{8}$ More importantly, one can consider the question if it is better to have a less stable calculation or is it better to neglect the tail all together as in a $\mathrm{VaR}$ calculation. We believe that the answer to that question is obvious and in favour of $E S$.

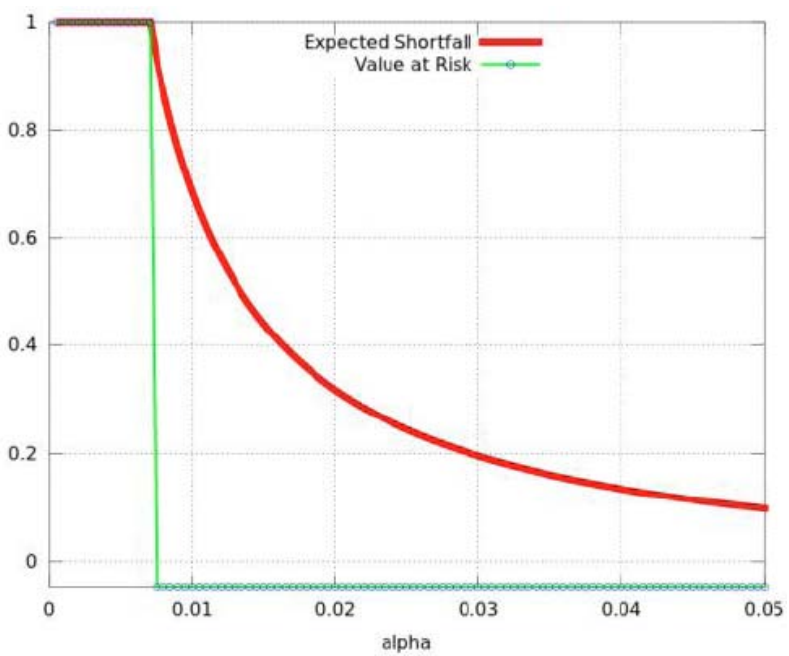

Figure 2: The $\operatorname{VaR}_{0.01}$ and $E S_{0.01}$ in function of $\alpha$ for one bond as defined in Example 2.1. If one believes that $V a R$ is reasonable, then it is reasonable to see the risk drop from $100 \%$ to $-5 \%$ when the desired confidence level passes from $(1-0.0069999)$ to $(1-0.0070000)$. It is reassuring that $E S$ is continuous in $\alpha$.

\section{Spectral Risk Measures}

A simple and elegant proof for sub-additivity of $E S$ for discrete distributions is presented by De Brouwer (2012) [20] (p. 125). For continuous distributions the proof is generally given via Distortion Risk Measures or via Spectral Risk Measures. Both representations are equivalent. follows.

In general, we can define spectral risk measures as

\section{Definition (Spectral Risk Measure)}

Let $X$ be a stochastic variable, representing the return of a financial

\footnotetext{
${ }^{8}$ For portfolio optimization and risk minimization we refer to (see for the fundamentals: Rockafellar and Uryasev, 2000 [34], 2002 [35]; Uryasev, 2000 [40]; Pflug, 2000 [18]) and (for overviews: De Brouwer, 2012 [20]; Acerbi and Simonetti 2002 [25]; Strepparava, 2009 [37]); for comonotonic approximations: (see Vanduffel, 2005 [41]; Vanduffel, Chen, Dhaene, Goovaerts, Henrard, and Kaas, 2008 [42]; Dhaene, Vanduffel, and Goovaerts, 2007 [29]; Dhaene, Vanduffel, Goovaerts, Kaas, an Vyncke, 2005 [30]; Vanduffel, Hoedemakers, and Dhaene, 2005 [43])
}

asset. Then we define the spectral measure of risk $M_{\phi}(X)$ with spectrum (or risk aversion function) $\phi(p):[0,1] \mapsto \mathbb{R}$ as:

$$
M_{\phi}(X):-\int_{0}^{1} \phi(p) F_{X}^{\leftarrow}(p) d p
$$

Example 2.4. The spectrum or risk aversion function for the $\alpha$-Expected Shortfall $\left(E S_{\alpha}\right)$-as defined in next section-is

$\phi_{E S_{\alpha}}(p)=\frac{1}{\alpha} \mathbf{1}_{[p \leq \alpha]}:=-\left\{\begin{array}{l}\frac{1}{\alpha} \text { if } p \leq \alpha \\ 0 \text { else }\end{array}\right.$

Example 2.5. The spectrum or risk aversion function for the $\alpha-V a R$ is the Dirac delta function:

$\phi_{\operatorname{VaR}_{\alpha}}(p)=\delta(p-\alpha)$

The spectral representation of risk measures clarifies a lot. $^{9}$ Via this presentation it is possible to determine necessary and sufficient conditions on the spectrum for a risk measure to be coherent.

Theorem 2.2. The risk measure $M_{\phi}(X)$ as defined above is coherent, if and only if

$$
\left\{\begin{array}{l}
\phi(p) \text { is positive } \\
\phi(p) \text { is not increasing } \\
\int_{0}^{1} \phi(p) d p=1
\end{array}\right.
$$

Proof. (see Acerbi (2002) [21] )

This theorem proves that there is a deep relation between what we have defined as "coherent" and a non-increasing risk spectrum. A not-increasing risk spectrum means that calculating the risk measure, one cannot assign a lower weight to a worse outcome. In other words the spectrum $\phi(p)$ of the risk measure $M_{\phi}$ determines the weights associated to possible outcomes. This explains the alternative name for $\phi(p)$ : "risk aversion function".

A person who thinks coherently cannot allocate a higher weight to better outcomes in a risk measure, and hence the risk aversions function is not increasing. And this is what $V a R$ does: it assigns a zero weight to all outcomes worse than a certain quantile: in Equation

\footnotetext{
${ }^{9}$ More details can for example be found in De Brouwer (2012) [20], Acerbi
} (2004) [17] and most of the proofs are in Acerbi (2002) [21] 
3 one can see that the spectrum of $V a R$ increases infinitely steep just before $\alpha$.

\section{Portfolio Optimization and Risk Minimization}

A first area where we will illustrate the potential devastating impact of using incoherent risk measurements is the construction of an investment portfolio of assets.

\section{Value at Risk ( $\mathrm{VaR})$}

Example 3.1. Consider the risk surface (efficient frontier) of Example 2.3.
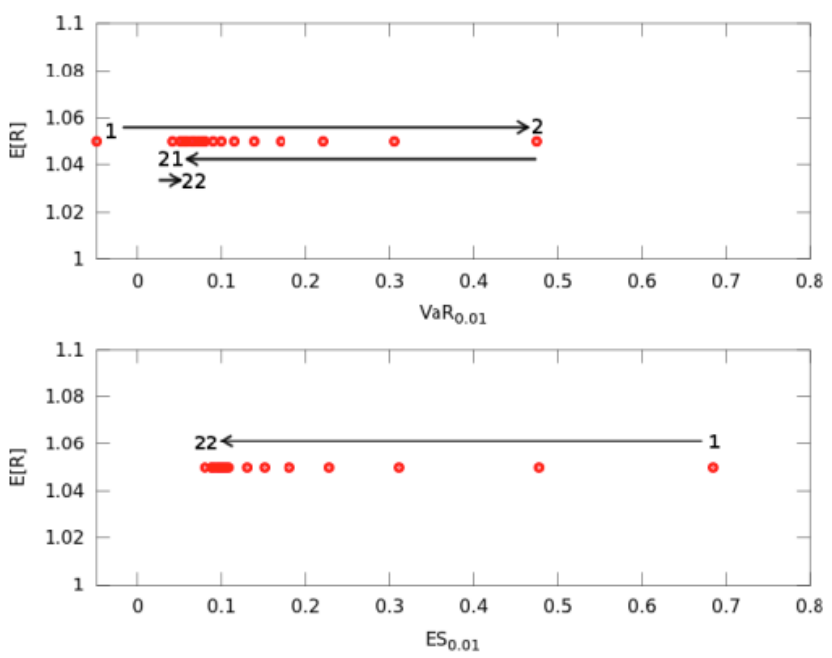

Figure 3: The risk surface of the $\operatorname{VaR}_{0.01}$ and $E S_{0.01}$ of Example 3.1. The numbers-next to the dots-correspond to the number of independent bonds in portfolio. ES will decrease as diversification increases, $V a R$ behaves unpredictable in function of diversification.

A numeric optimization using $V a R$ will converge to a local minimum, such as one, 21 or 63 bonds. When a coherent risk measure, such as $E S$, is used the "most safe" portfolio is the one with best attainable diversification. Refer to Figures $\mathbf{1}$ and $\mathbf{3}$ for two different views on this problem.

Also here we see that a coherent risk measure finds a logical answer, an incoherent risk measure can and will give rise to surprises.

\section{Variance (VAR)}

As mentioned in the introduction, Markowitz (1952a) [5] introduced the riskreward optimization and used variance as first proxy for risk. Variance is defined as follows.

\section{Definition (Variance (VAR))}

Let $X$ be a real-valued stochastic variable, its variance is then defined as:

$$
\begin{aligned}
V A R & :=E\left[(X-E[X])^{2}\right] \\
& =E\left[X^{2}\right]-(E[X])^{2} \\
& =\int_{\mathbb{R}}(x-\mu)^{2} f_{X}(x) d x
\end{aligned}
$$

with $\mu:=E[X]=\int_{\mathbb{R}} x f_{X}(x) d x$.

Note that since standard deviation is a monotonous function of $V A R$ that all the results in this paper that are obtained for $V A R$ are also valid for standard deviation.

Example 3.2. Consider a portfolio construction problem for an investor who has the choice between the assets presented above in Figure $\mathbf{4}$ (more details in Appendix A), who aims to minimize risk on a one year horizon. The results of this portfolio optimization problem can be found in Figure 5. An investor that wants to minimize risk on a one year horizon will find a well diversified portfolio with a mean-variance approach, but will face a significant probability to loose money (17\%). The investor who uses a coherent risk measure -and minimizes risk- will invest all in the structure with capital protection ${ }^{10}$ and hence have a zero probability to loose money.

The results for the optimization problem defined by Example 3.2 are presented in Figure 5.

If one prefers the most safe portfolio, then-in this example-it would be logical to invest only in the structured fund with capital guarantee, because that is the only investment that a zero probability to loose money. The minimum ES portfolio is indeed this logical solution. The minimum variance portfolio however is better diversified, but has an important probability to loose money.

Another remarkable issue is that the variance optimization allocates very fast about one third to the hedge fund that has a significant probability to loose half of the invested money. Also this is not logical, one would expect the exposure to the hedge fund to increase slowly (from zero for the most defensive portfolio). That is indeed the result found with meanES optimization.

Once more the same conclusion imposes itself: a coherent risk measure finds results that are logical and conform our intuition, an incoherent risk measure can and will be "fooled".

\footnotetext{
${ }^{10}$ The structure with captial protection can be seen as a portfolio that holds a zero bond and a long call option. The zero bond is chosen so that at maturity the value equals the initial captial, so the investor will get his/her money back even in an adverse market where the option expires without value.
} 

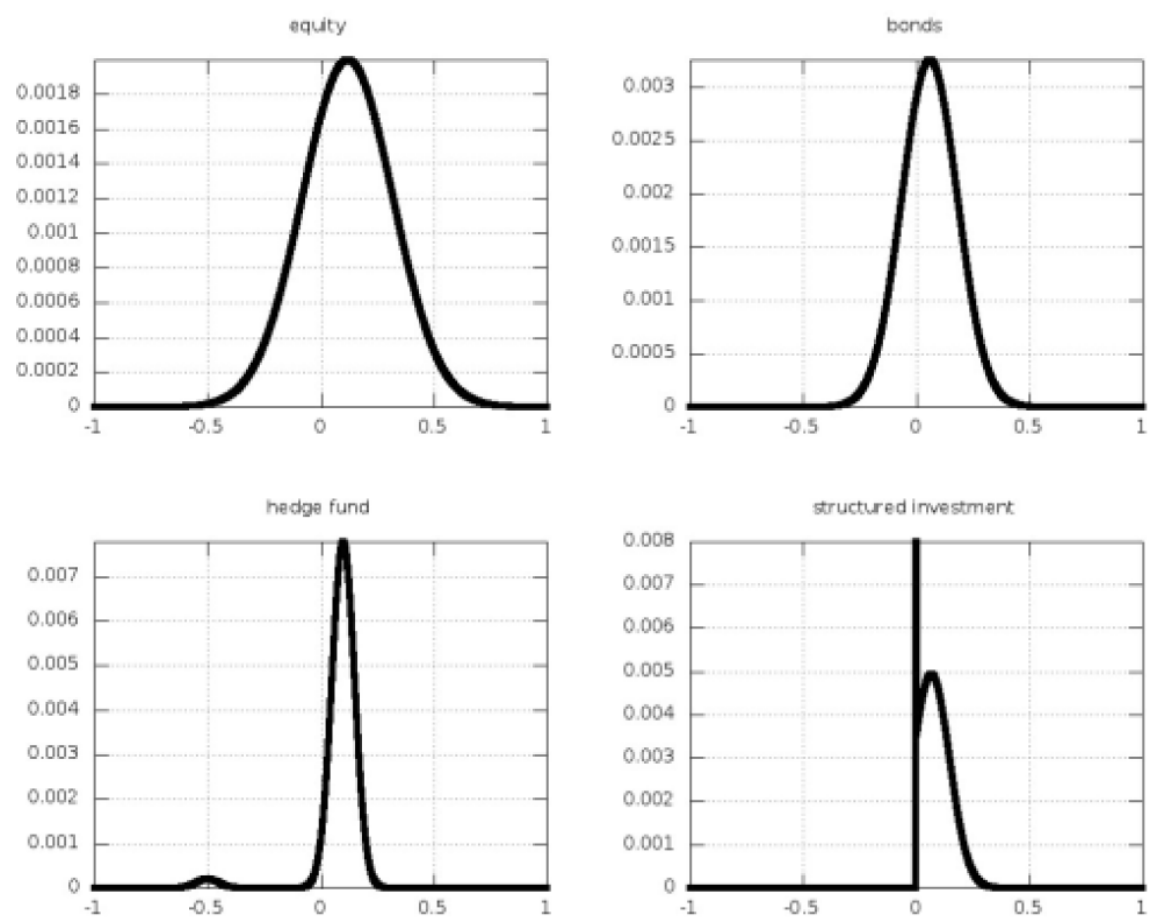

Figure 4: An overview of the probability density functions of the assets used in the examples. Note that the $y$-axis of the last graph (for the structured investments) is truncated.
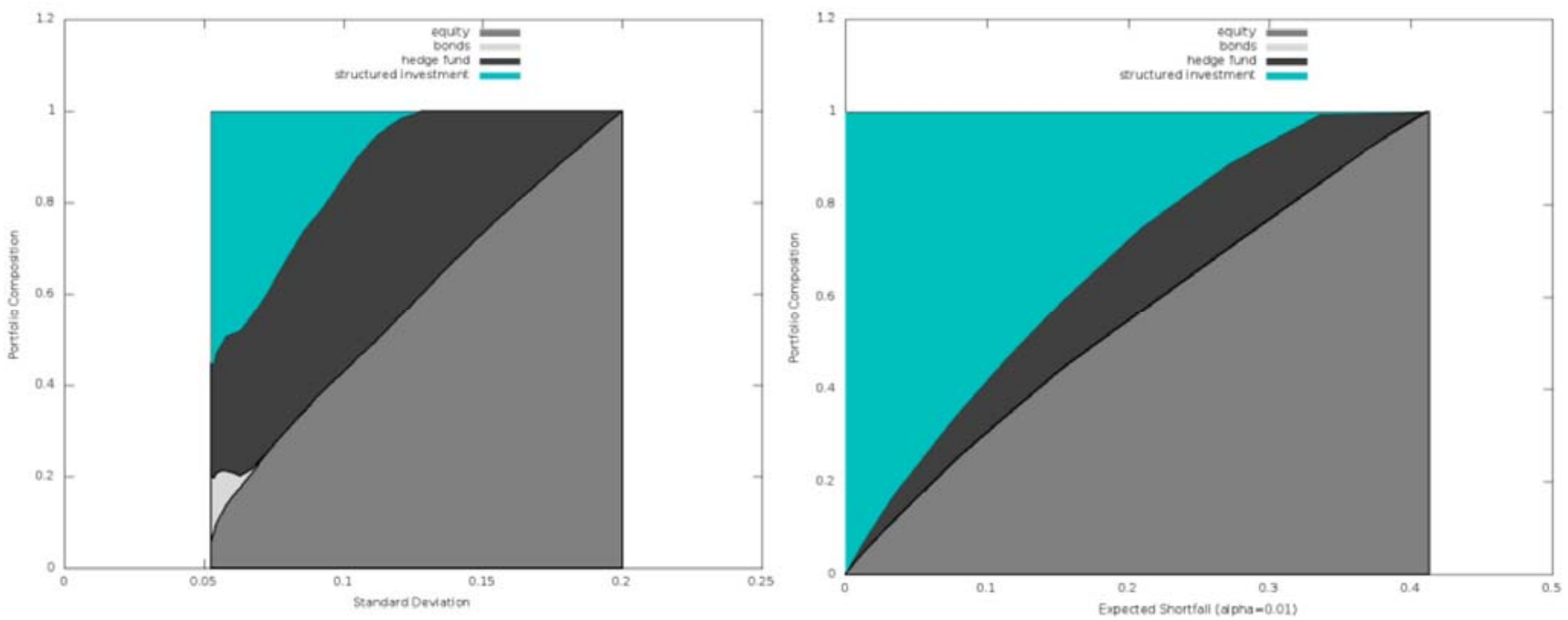

Figure 5: The optimal portfolio compositions for Example 3.2. Above for the mean-varianceoptimization and below for a meanexpected shortfall optimization.

\section{REGULATORY USE OF RISK MEASURES}

\section{Using VaR as a Risk Limit}

In this section we study the UCITS regulation. UCITS is short for Undertaking for Collective Investments in Transferable Securities. This legislation aims to create a framework for mutual funds in the European Markets and on top of that ensures a level playing field and even introduces a passport system for investment funds. These developments are a great opportunity for international market players, but the aim was also to "ensure investor protection" (EU Directive 2004/383/EC of 27 April 2004-second point in the opening). In stead of looking for a coherent riskmeasure, they established "an extensive system of risk-limitation". The intention was to open up the possibility for UCITS funds to invest in modern financial instruments such a derivatives, but at the same time to keep the risks under control. 
The Council Directive 85/611/EEC of 20 December 1985 (as subsequently amended by Directive 2001/108/EC) provides in Article 19.3 that "A UCITS shall ensure that its global exposure relating to derivative instruments does not exceed the total net value of its portfolio."

The Commission Recommendation 2004/383/EC further explains its approach in point three. It recommends that "non-sophisticated UCITS" use a commitment approach, which is basically the equivalent underlying position in primary assets (for an option this would be the delta-as explained in point 3.2.2 of 2004/383/EC). For "sophisticated UCITS" member states are recommended to use a "VaR approach" (point 3.3.1) and the Commission explains: "In the VaR approach, the maximum potential loss that a UCITS portfolio could suffer within a certain time horizon and a certain degree of confidence is estimated." The careful reader will appreciate that VaR does exactly the opposite of what is explained here: $V a R$ estimates the minimal loss to be expected in the $100 \alpha \%$ worst cases! $!^{11}$

The details of the implementation are further laid out by CESR (now ESMA: the European Securities and Markets Authority) CESR produced its General Guidelines for calculating the global exposure in 2010 (document reference CESR/10-788) a call for evidence was done for these Guidelines (CESR/10-1253) and a final report (ESMA/2011/112) summarizes this call for evidence. Unfortunately the feedback about the use of an incoherent risk measure (see De Brouwer, 2010 [22]) was not reflected in this overview.

In CESR/10-788 VaR is presented as a method "to calculate global exposure" (point 1.box-1.4 - pg. 5), however the same sentence calls it also "an advanced risk measurement methodology". Both statements are rather odd for two reasons. Firstly if "global exposure" is assumed to be the root of all evil, then how is that equivalent to $V a R$ ? Secondly it is not coherent to call $\operatorname{VaR}$ a "risk measurement methodology" and it is misleading to call it "advanced", especially 13 years after Artzner, Delbaen, Eber, and Heath (1997)'s [1] paper.

\footnotetext{
${ }^{11}$ This underlines how deceiving $V a R$ is, the apparent simple interpretation has to be stated very carefully. This wrong and misleading information survived in the European regulations to date, and worse, it has been copied in local texts. See for example Central Bank of Ireland (2011) [28], chapter 3 - page 15. In that booklet $V a R$ also is wrongly explained to "measure [note that "estimate" is replaced by "measure"] the maximum potential loss at a given confidence level (probability) over a specific time period under normal market conditions". Besides the mixing up of "lowest" with "maximum", the undefined notion of "normal market conditions" is added. This is most remarkable, because wouldn't we expect a risk measure to forecast "abnormal market situations"? Fortunately the next paragraph in the booklet provides an example contradicting the paragraph defining $V a R$. This paragraph makes clear that $V a R$ is a proxy for the smallest loss to expect with a confidence level of $(1-\alpha)$
}

The UCITS directive proposes to limit risk via limiting "global exposure". This concept is rather elusive, not universal, only one of many causes of risk and therefore not a risk measure at all. If ESMA is to make sense out of this, then it can be useful to replace an incomplete concept with a universal risk measure. Unfortunately, the incoherent risk measure $V a R$ is chosen.

So, besides the "commitment approach", CESR/10788 proposes two different $\mathrm{VaR}$ methodologies that both are assumed to do the same thing (but lead to different results): the Relative $V a R$ method and the Absolute VaR method. ${ }^{12}$

First there is the "Relative VaR method" (typically for funds that are managed relative to a benchmark). A $1 \% \mathrm{VaR}$ has to be calculated on monthly data that spans a year; and then annualized with the square root law (see Appendix B). The "risk limit" for "relative VaR" is defined as follows.

$$
\frac{V a R_{U C I T S}-V_{\text {RaferencePorfolio }}}{V_{\text {ReferencePorfolio }}} \times 100 \leq 10 \%
$$

Besides the fact that on the left side the percentage sign is omitted $^{13}$, this definition states that a benchmarked portfolio should be close to its benchmark... whatever that benchmark is. This is a very peculiar approach to "risk", but it is in accordance with 2004/383/EC. We struggle however to see how this can be a sensible risk measure.

Second, there is the "Absolute VaR" for UCITS that are not managed relative to a benchmark, and the limit is defined as follows.

$\operatorname{VaR}_{\text {UCITS }} \leq 20 \% N A V$

Where $N A V$ refers to the net asset value of the UCITS.

The fact that hereby ESMA goes beyond the proposition of 2004/383/EC, and does not focus on one aspect (exposure) but makes and attempt to identify the more fundamental problem and uses a risk measure in the rule from Equation 8 is laudable.

\footnotetext{
${ }^{12}$ Note that there is a quadruple layer of cognitive dissonance in this legislation: (1) using "global exposure" as a proxy for all risk, (2) assuming that it is equivalent with $\operatorname{VaR},(3)$ assuming that the commitment approach and the $V a R$ approach are equivalent and (4) assuming that the Relative $V a R$ and the Absolute $V a R$ measure the same. If they would not be assumed to be the same, then they would not be equivalent and hence in contradiction with $85 / 611 / E C ' s$ requirement. We argue that this is related to the choice for an incoherent risk measure. If one would have chosen a coherent risk measure, then one measure with one limit would $t$ all cases and no exceptions would be needed.

${ }^{13}$ This mistake was also reported in De Brouwer (2010) [22], but to date it hasn't been corrected. This mistake makes this rule to limit the relative $V a R$ to $0.1 \%$ in stead of the intended $10 \%$.
} 
However this is fundamentally different from the rule expressed in Equation 7, that focusses only on one risk contributor: exposure. Another interesting paradox is that CESR/10-788 requires daily calculation of the "risk measure". This is strange because investors buy these products and hold them for many years. If the risk measure can change on a day to day basis then it is useless for the investor. Fortunately the "risk measure" constructed will not change daily because it is the average over at least 250 observations. A check every month would be a lesser burden to the industry and lead to similar results. More importantly, a risk measure-for investors - should be stable, because it should forecast problems (and not be a measure for the recent past). If however we assume that we want a measure that adapts to the recent past, then it would have made more sense to suggest for example an exponential weighting of observations in stead of a daily calculation.

Let us now consider a simple example.

Example 4.1. Consider a structured fund that will pay on one year time $105 \%$ of the initial investment (assume that it pays the capital back plus a coupon of $5 \%$ in one year), except if company $X$ defaults in that year, then it pays $0 \%$. We estimate the probability that company $X$ defaults in one year to equal $0.7 \%$.

The $V a R_{\text {UCITS }}$ is $-5 \%$, so this is perfectly acceptable according to the General Guidelines of CESR/10-788.

Example 4.2. Consider a structured fund that will pay on one year time $105 \%$ of the initial investment, if either company $X$ or $Y$ defaults then it pays $52.5 \%$ of the initial investment, and if both companies $X$ and $Y$ default then it pays zero. We estimate the default probability of both company $X$ and $Y$ to equal $0.7 \%$, and their default possibility is independently distributed. The $V_{a R_{U C I T S}}$ is $47.5 \%$, so this is not acceptable according to the General Guidelines of CESR/10-788.

While the fund from Example 4.2 would be a lot more safe and better diversified that the one from Example 4.1, $\operatorname{VaR}$ will erroneously mark it as more risky. Once more we find that the incoherent risk measure provides an absurd result, and that the coherent risk measure, $E S$, does live up to our expectations. The ES for Example 4.1 would be $68.5 \%$ and in the better diversified fund from Example 4.2 it would be reduced to $47.5 \%$.

One will recognize here the example of previous section. Again we see that because VaR is not subadditive, it can wrongly indicate the better diversified portfolio as more risky. $\mathrm{VaR}$ is incoherent and cannot be used in regulation, certainly not at the level of individual funds!
Another problem is the cognitive dissonance that arises from the fact that three different methods are assumed to measure the same but are totally different and not even additive.

Hundreds of pages of regulations, thousands of people involved in designing and implementing a complex legislation, that ultimately misses its goal could be replaced by one simple rule: "A UCITS funds shall keep its $\alpha$-Expected Shortfall on a $T$ year horizon lower than $\mathrm{x} \%$ of the $N A V$. Funds with fixed maturity (among others, structured funds), however, calculate their Expected Shortfall only at the start and with a horizon matching the lifetime of the UCITS and must keep this ES below y\% of the $N A V . "$

This simple rule would in contrast with the existing regulation

1. be clear and simple: it would not require hundreds of pages to explain. It would simply require some general principles on how to calculate the ES (similar to the rules that are now used to calculate VaR); but more importantly there would be no need for loads of secondary definitions and special rules;

2. be reliable: it would really mean something for the investor because a coherent risk measure will lead to coherent results;

3. create the possibility to link this limit with a risk classification at product level (wouldn't it be logical that the upper boundary of the highest risk class is also the risk limit?).

Disregarding scientific research in law-making costs much money and leads to crippled regulation that is full of loopholes and finally provides no coherent information for the investor. In this case it encourages a product design that favours large but improbable losses and less diversified portfolios. The reader will appreciate here the similarities with the course of events during the Global Meltdown in 2008.

The authors appreciate the efforts of law makers to keep UCITS a quality label and believe together with the law-makers that part of this quality label is the characteristic "not too risky". We argue that this notion "not too risky"14 can be expressed as a limit on one coherent risk measure, it would avoid a myriad of confusing concepts such as "complicated UCITS" or

\footnotetext{
${ }^{14}$ The UCITS label guarantees quality on procedures, control, custody, administration, etc. besides an attempt to keep the investments from being too risky. The efforts are laudable and the intentions noble. However, the failure to select a coherent risk measure|seven years after the axioms had been written and published|resulted in a crippled legislation that adds a huge burden on financial regulators and for the whole industry while it fails to provide real protection for the investor and is confusing.
} 
"not complicated UCITS", "commitment approach", "relative VaR", "absolute VaR", "global exposure" or "risk exposure", etc. All these concepts are only derivatives of what it is really about, and are unnecessary complications. This is similar to an effort to ban riots by forbidding guns, then masks, then meetings, then newspapers, etc.... while the underlying-real-problem is not addressed. In such case we're set for a race to increase legislation that is bound not to meet its target.

\section{A Risk-Reward Indicator Based on Volatility}

On July 1st, 2010 CESR proposed-still in the framework of the previously mentioned UCITS IV regulation-to create a "risk and reward indicator" based on volatility (in document with ref CESR/10-673). The risk reward indicator is an interesting idea. Its aim is to create an indication of risk for all UCITS (but if chosen carefully, it could have been used for all investments). If one disregards the investment goal, such parameter can be a useful indication for the risk of an investment. ${ }^{15}$

This idea was mentioned in the Commission Regulation 585/2010 of 1 July 2010 and repeated in the Directive 2009/65/EC in very broad terms. Article 78.31.e of the Directive states that the Key Investor Information should contain a "risk/reward profile of the investments" ${ }^{\text {"16 }}$. CESR then endeavoured to write implementation measures (see CESR/09-1026) and published it's guidelines for the risk-reward indicator as CESR/10-673 on 1 July 2010. These guidelines provide the information that the risk-reward indicator has to be based on volatility, and provide further details on how to calculate the volatility. The volatility is defined as a standard deviation of weekly returns (but annualized with the square root $\operatorname{law}^{17}$ ).

The regulation further presents provisions about what to do if the fund has or has not 5 year of history, and finally the number found is scaled back to an ordinal scale via an arbitrary scaling. (box 2, pg. 7 in CESR/10-673)—see Table 1.

The reason for scaling down the risk measure to an ordinal scale could be to mask imperfections, improve apparent stability, and reduce expectations of precision

\footnotetext{
${ }^{15}$ Recent research rather indicates that a risk indication at the level of the investment is rather misleading, as the true risk is inherently linked to the investment goal and the other holdings in a non-trivial way. See for example Amenc, Martellini, Milhau, and Ziemann (2009) [13] and De Brouwer (2011) [12]. This would make a risk class at the level of an investment a loose concept that has to be handled with much care.

${ }^{16} \mathrm{~A}$ profile seems to be a much richer and multidi-mensional concept than an indicator (that is typically one number).

${ }^{17}$ Also here one can strictly speaking not use the square root law. However the problem is not as big as in the previous example, it is a monotonous transformation and in the next step|labelling of risk classes|it would anyhow be reduced to a ordinal scale. See Appendix B for some background on the square-root-law.
}

that it might otherwise create among investors. This is wise, as it is particularly difficult to predict financial markets. This scale introduces however seven additionally parameters (the borders of each riskclass), where this could be reduced to two parameters (the upper limit and the number of classes), with a simple logical reasoning (see De Brouwer, 1998 [23]), however this is an academic issue as such scaling reduces the risk class anyhow to an ordinal scale.

Table 1: The "Risk Classes" as Defined by CESR in CESR/10-673, pg. 7, in the same Document the Risk Classes are also Referred to as "Risk and Reward Indicator"

\begin{tabular}{|c|c|c|}
\hline risk class & volatility equal or above & volatility less than \\
\hline \hline 1 & $0 \%$ & $0.5 \%$ \\
\hline 2 & $0.5 \%$ & $2.0 \%$ \\
\hline 3 & $2.0 \%$ & $5.0 \%$ \\
\hline 4 & $5.0 \%$ & $10.0 \%$ \\
\hline 5 & $10.0 \%$ & $15.0 \%$ \\
\hline 6 & $15.0 \%$ & $25.0 \%$ \\
\hline 7 & $25.0 \%$ & $+\infty$ \\
\hline
\end{tabular}

Two remarks impose themselves here. First, even CESR (ESMA) uses in the table the wording "risk class". This is more modest and closer to the reality than "risk and rewards indicator", that concept is ill defined and only makes sense in very restricting - and arguably wrong-assumptions about financial markets. Second, the use of numbers as labels for an ordinal scale is very disturbing as it creates the false impression for the untrained investor that this is a ratio scale. This is misleading as the aim is to build an ordinal scale. The obvious solution would be to use labels such as A, B, C, etc. (see De Brouwer, 1998 [23]).

A little beyond these two obvious remarks, one will notice that a "risk class" based on an incoherent risk measure is no risk classification at all, it is not even an ordinal scale, but a meaningless nominal scale. To understand this we propose to recall the example that has been used before and calculate the risk classes associated to them.

Example 4.3. Assume the assets from Example 3.2 plus one "risky bond" (this could also be a structured fund based on a digital option) that has a probability of $1 \%$ to loose $15 \%$ and a probability of $99 \%$ to gain $5 \%$. Then consider the risk class as defined by CESR/10673. The results are as in Table 2.

The example above indicates the weakness of using variance (or volatility) as underlying risk measure for any classification. Expected shortfall $(E S)$ on the 
other hand is a coherent risk measure and the results are reasonable and consistent with reasonable expectations.

Table 2: The risk classes for Example 4.3. CESR/ESMA's method considers the hedge fund that has roughly a $2.5 \%$ probability of loosing about $50 \%$ of its value is in the same risk class as a bond fund. A structured fund that has no risk to lose something ends up in the fourth risk class, but the risky bond that has a $1 \%$ probability of loosing $15 \%$ is considered as very safe!

\begin{tabular}{|c|c|c|c|}
\hline Portfolio & risk class & $\boldsymbol{\sigma}$ & $\boldsymbol{E} \boldsymbol{S}_{\mathbf{0 . 0 1}}$ \\
\hline \hline Equity & 6 & 0.2000 & 0.4123 \\
\hline Bonds & 5 & 0.1200 & 0.2660 \\
\hline hedge fund & 5 & 0.1062 & 0.5482 \\
\hline structured investment & 4 & 0.0671 & 0.0000 \\
\hline risky bond & 2 & 0.0198 & 0.1500 \\
\hline mix $1 / 2$ equity $+1 / 2$ bonds & 5 & 0.1173 & 0.2223 \\
\hline
\end{tabular}

These simple and realistic ${ }^{18}$ examples make clear how absurd the results of this volatility based risk classification will be. If one would be less ambitious and focus only on risk (not reward) and choose a coherent risk measure, the results would be logical and be useful. Failing to select a coherent risk measure underlying the risk classification made the method useless. This will now result in additional explanations, footnotes and disclaimers, leaving the investors more confused.

We provide below an overview of the goals set out in CESR/10-673, and try to asses how well the goals are met with the existing legislation.

1. provide investors with a meaningful indication of the overall risk and reward profile of the UCITS

It is misleading to talk about a risk and reward indicator and then base it solely on a "risk measure". The risk measure chosen is not a coherent one, hence the method can and will yield wrong and counter-intuitive results (as shown above). Also standard deviation is not an intuitively understandable concept. Additionally, the classification is based on arbitrary windows of volatility (see Table 1), which introduces

\footnotetext{
${ }^{18}$ It would have been easier to assume an assest with an expected return of $100 \%$ and a volatility of $25 \%$ (risk class 7 , while it would be an excellent and safe investment in all possible coherent ways of thinking about investments) and compare this with an asset that has an expected return of $-100 \%$ with a volatility of $0 \%$ (risk class 1 ). Such examples also indicate that the method is flawed, but these assets would not realistically be available. The assets from our examples are marked-to-market in some definition. Note that also in these examples ES would provide logically coherent results.
}

unnecessary arbitrary parameters that reduce in itself the meaningfulness of the method. As icing on the cake a numerical scale is used, which will be misleading to investors, who will assume automatically that it is a ratio scale, where-in fact-it is a meaningless nominal scale. A coherent risk measure could bring this up to an ratio scale. However, it would be prudent to promote it as an ordinal scale.

2. ensure appropriate spread of UCITS across different risk classes

This should not be a goal, otherwise it creates circular reasoning. However we understand that in absence of logical principles, this goal gives false comfort that the method chosen is "good" in some sense. In reality, this is not necessary (wouldn't it be interesting to find out if the investments offered are evenly spread among real risk classes?) and leads to circular reasoning.

3. be applicable to all types of UCITS

Indeed, this is possible. However for UCITS, that have a distribution deviating from a bell-shape, it has no reliable interpretation with relation to risk.

4. leave no room for manipulation

As we have shown, an incoherent risk measure is bound to be open for manipulation. See the examples in this paper.

5. enable easy and cost-effective implementation by UCITS providers

This goal is archived.

6. be easily understood by auditors, advisers and distributors

The method is simple enough, however is it truly understandable? Does someone understand what $V a R$ means in terms of risk? In other words: what are the underlying axioms that would classify $V a R$ as a coherent risk measure?

7. enable easy and effective supervision by regulators

This goal is achieved.

8. achieve an adequate degree of stability in the risk classification process with respect to normal trends and fluctuations of financial markets

This goal is achieved, but should a risk measure not give an indication about bad times, and should it hence not be relatively stable even in bad times?

Once more we're bound to conclude that using an incoherent risk measure inevitably leads to absurd situations. We are convinced that law-making shouldwhere possible-be based on scientific literature. 
Especially rule-based regulations could otherwise inadvertently lead to dangerous and misleading situations. In this case, consulting just a few papers would have avoided much of the disarray. Artzner, Delbaen, Eber, and Heath (1997) [1] would have pointed us to the importance of using a coherent risk measure, Pflug (2000) [18] would have provided a coherent risk measure ${ }^{19}$ and De Brouwer (1998) [23] would have added suggestions to avoid pitfalls such as using numerical labels for an ordinal scale, limiting the number of assumptions and arbitrary parameters and hence increasing internal coherence and constancy.

Also the difference between the risk limit, based on $V a R$, and the risk classification, based on standard deviation, points towards some form of cognitive dissonance and can give rise to examples of investments that would not be possible because of the risk limit but have a low risk class.

Example 4.4. A simple example is a structured fund that offers a $1 \%$ probability to loose $21 \%$ and a $99 \%$ probability to gain $5 \%$. Such fund would not be allowed to trade, because its $1 \% V_{\text {VaR }}$ UCITS would be $21 \%$ (exceeding the limit and being logically classified as "too risky"). Its volatility is $2.5870 \%$, that is only risk class 3 , hence considered as safer than bonds-from our example, in the middle of the spectrum, and perfectly acceptable.

If one would want to reduce the ESMA-risk-class of the fund from Example 4.4, one is encouraged to increase the management fee so that the pay-off becomes: $1 \%$ chance to loose $21 \%$ and a $99 \%$ chance to loose $16 \%$...the risk class drops then to the lowest risk class! Also this is not possible with a coherent risk measure, increasing the management fee will increase a coherent risk measure or at least keep it stable ${ }^{20}-$ because of the monotonicity axiom.

One coherent risk measure could cover both risk limit and risk classification so that both would be congruent; and would not lead to cognitive dissonance as in the example above. But more importantly it would not be possible to manipulate the results so easily.

\section{CONCLUSIONS}

We already mentioned that the title of this paper is a reference the seminal paper of Artzner, Delbaen, Eber,

\footnotetext{
${ }^{19}$ Both paper scan be replaced by the-more accessible for nonmathematicians-paper Acerbi (2004) [17]. Additionally this paper is highly recommended for correcting some definitions from Pflug (2000) [18] and it uses a terminology that will be more familiar for asset managers: it uses profit variables (in stead of loss distributions as customary in actuaria papers).

${ }^{20}$ Note that in this example $1 \%$ - ES would be stable, because we manipulate only the pay off sabove the $1 \%$ cutoff. This phenomen on is related to Important Notice number 3 at the end of this document.
}

and Heath (1997): "Thinking Coherently" [1], however with hindsight it might also be a reference to Oscar Wilde's play "The importance of being Earnest". We live in a world where our imagination plays an eminent role. Since 1998 Artzner, Delbaen, Eber, and Heath (1999) [24] we know that $V a R$ is not coherent, but we choose to ignore that reality; and both practitioners and regulators embrace more and more $V a R$.

This paper shows how dangerous this can be in the setting of a personal portfolio. But also for financial institutions and even banks and countries the same results will hold. VaR can and will lead to incoherent indications about risk!

$\mathrm{VaR}$ is not an acceptable (or coherent) risk measure because it disregards the tail of the distribution completely. Big financial crises show us that the devil dwells in the left tail of the distribution. The computational problems (more computationally intensive and less stable results) seem-to us-no excuse to completely disregard the tail of the distribution. Only when using a coherent risk measure, one will obtain coherent results.

Also the popular standard deviation is only a measure for dispersion, not a measure for risk. This is because standard deviation and variance attribute an increasing contribution to better outcomes, which is not logical for a risk measure to do.

By constructing examples, we have proven that both $V a R$ and $V A R$ are incoherent risk measures-not only in a mathematical sense but also in a semantic sense-and hence can and will lead to illogical results. By extension, any method based upon them is inconsistent and flawed. Markowitz (1991) [8] remarked that "Semi-variance seems more plausible than variance as a measure of risk, since it is concerned only with adverse deviations" (p. 286); and since Artzner, Delbaen, Eber, and Heath (1997) [1] there is proof that his insight is a deep truth. The examples presented in this paper clearly show that coherence matters not only in the minds of mathematicians, but also in the portfolio selection in real life.

\section{IMPORTANT NOTICES}

1. It is not the author's intention to undermine the work done for UCITS IV. UCITS IV is a good legislation, with good intentions; and indeed the UCITS regulation is so much more than only a $V a R$ limit and a VAR based risk-class. However, in its implementation is one crucial mistake: the choice for two incoherent risk measures ( $\mathrm{VaR}$ and standard deviation) and this has immense consequences. 
2. Please note that also a coherent risk measure is also just one view via one frame ${ }^{21}$ on the complex reality of the modern financial markets. Even a view through a "coherent window" necessarily reduces the multidimensional, complex and interlinked reality to $\mathbb{R}$. This is always a reduction of the true richness of the dangers on financial markets, necessarily based on information from the past and it will never replace risk management by humans, nor will it prevent (previously unseen) adverse moves in financial markets!

3. Even a coherent risk measure is only a risk measure and not a "risk and reward indicator".

Also note that illustrations, Figures 1, 2, 3, 4 and 5 also appeared in the author's doctorate - see De Brouwer (2012) [20] - all rights reserved by the author.

\section{NOMENCLATURE}

[. $]^{+} \quad \max \{0,$.

$\alpha \quad$ a level of probability, $\alpha \in[0,1]$ (to characterize the tail risk $\alpha$ will be "small"for example for a continuous distribution one can say with a confidence level of $(1-\alpha)$ that the stochastic variable in an experiment will be higher than the $\alpha$ quantile)

$\mu \quad$ the vector of the expected values of the different assets

$\boldsymbol{R} \quad$ the stochastic vector of returns

$\delta($.$) \quad the Dirac Delta function$

$\delta(x-a)=\left\{\begin{array}{c}0 \text { if } x \neq a \\ +\infty \text { if } x=a\end{array}\right.$,

but so that $\int_{-\infty}^{+\infty} \delta(x-a) d x=1$
${ }^{21}$ "Frame" should be understood as in the sense of a behaviouralbias as described by Tversky and Kahneman (1981) [39].

${ }^{22} E S$ is consistent with second order stochastic dominance (see Yamai and Yoshiba, 2002a [45], p. 109), but this only means that if stochastic variables are ordered by stochastic dominance of the second order, then this implies the ranking of the $E S$. The opposite is not true, a Lower $E S_{\alpha}$. is not a suffcient condition for stochastic dominance! In other words, $E S_{\alpha}$ is blind for events that are above the $100 \alpha \%$-cut-off and these can influence stochastic dominance of the second order (and hence preference, because second order stochastic dominancie is a suffcient condition for expected utility preference for risk averse investors who prefer more over less). Only the infinite set of all possibile $E S_{\alpha}$. would be a suffcient condition for second order stochastic dominance. We believe that it is safe to conjecture that it is not possible to reduce both risk and reward to $\mathbb{R}$ in a coherent way, and hence it is misleading for investors to use the terminology "risk and reward indicator".
$\Lambda$

$\mathbb{R}$

$\mathbb{V}$

$\mu$

$\phi(p))$

$\rho$

$\sigma$

$c$

$f_{X}(t)$

$f_{X}(x)$

$i$

$M_{\phi}(X)$

$p$

$q_{\alpha}(X)$

$Q_{X}(p)$

$R$

$R_{r e f}$

$R_{S F}$

$S$

$T_{i}$

X

\section{Leverage}

the set or real numbers

the set of real-valued stochastic variables

the average or expected value of a stochastic variable $X$

the risk spectrum (aka risk aversion function)

a risk measure

volatility $=$ standard deviation

the target return of an investment

the probability density function of a continuous distribution of a stochastic variable $X$

the cumulative distribution function of the stochastic variable $X$

Counter

a spectral risk measure

a probability (similar to $\alpha$ )

the $\alpha$-quantile of the stochastic $X$

the quantile function of the stochastic variable $X$

return on an investment

the return of the reference index

the return of the structured fund from the example

semi-variance

investment or time horizon with label $i$

a real valued stochastic variable, representing a profit variable

CERS Committee of European Securities Regulators, replaced on $1 / 1 / 2011$ by ESMA

DY Dividend Yield

ES Expected Shortfall

ESMA European Securities and Markets Authority

NAV Net Asset Value 
pdf probability density function

$V A R \quad$ Variance

VaR Value at Risk

\section{A BACKGROUND ABOUT THE EXAMPLE DISTRIBUTIONS}

We chose the following distributions.

$$
\begin{aligned}
& f_{\text {equities }}=f_{N(0.12,0.20)} \\
& f_{\text {bonds }}=f_{N(0.06,0.12)} \\
& f_{\text {hedge fund }}=0.025 f_{N(-0.50,0.05)}+0.975 f_{N(0.10,0.05)}
\end{aligned}
$$$$
f_{\text {structured fund }}(x)= \begin{cases}0 & \text { if } x<0 \\ \int_{-\infty}^{0} \frac{1}{\sqrt{2 \pi 0.20^{2}}} e^{\frac{(t-0.12-0.003)^{2}}{20.20^{2}}} d t & \text { if } x=0 \\ f_{N(0.12-0.03,0.20)} & \text { if } x>0\end{cases}
$$

Where $f_{N(\mu, \sigma)}$ denotes the pdf normal or Gaussian distribution with expected value $\mu$ and standard deviation $\sigma$.

From these distributions, it can be calculated that the expected return $\mu_{i}$ for each asset equals

$$
\mu=\left(\begin{array}{c}
\mu_{\text {equities }} \\
\mu_{\text {bonds }} \\
\mu_{\text {hedge fund }} \\
\mu_{\text {structured investment }}
\end{array}\right)=\left(\begin{array}{c}
0.12000 \\
0.06000 \\
0.08500 \\
0.07600
\end{array}\right)
$$

These expected values are-for the two base assets-close to their Black-Litterman estimate, calculated with equal market weights and without specific market view. ${ }^{23}$ The hedge fund has a very favourable sharp ratio, but there is a small probability $(2.5 \%)$ to loose about $50 \%$ of the investment. The Structured fund is constructed using a $2 \%$ interest rate combined with the volatilities resulting from the equities in the example in a Black and Scholes model for option pricing. It can be considered as a long call on the equity market combined with a deposit so that the deposit on one year time will yield exactly the initial investment. All this means that the pay-off of the structured investment is

\footnotetext{
${ }^{23}$ See Black and Litterman (1991 [26], 1992 [27]); or He and Litterman (1999) [31] and Satchell and Scowcroft (2000) [36] for more background.
}

$$
R_{S F}:=\Lambda\left[R_{r e f}-D Y\right]^{+}
$$

With $R_{S F}$ the return of the structured fund, $R_{\text {ref }}$ the return of the reference return-index, the leverage, $\Lambda$, at $40 \%$, and the dividend yield, DY, at $3 \%$.

In order to simplify calculations, but without decreasing the validity of the example, we assume that the five assets are independently distributed. This allows us to use convolutions in our calculations and we do not need perfect knowledge of the whole multivariate distribution.

\section{B THE SQUARE ROOT LAW}

The square root law states that one can estimate the volatility over a horizon $T_{2}$ based on the volatility over a period $T_{1}$ as follows: $\sigma_{2}=\sqrt{\frac{T_{2}}{T_{1}}} \sigma_{1}$. This only holds when returns are defined as additive quantities (for example log-returns) and when returns are independently distributed. Indeed, if returns are independently distributed, then the distribution of the total return can be found by convolving the individual return distributions; and variance is additive when variables are independently distributed (zero correlation).

For additive returns one has

$$
T_{1} R_{1}=T_{2} R_{2}
$$

with $R_{1}$ and $R_{2}$ the return over different time units, for example $R_{1}$ is the monthly return and $R_{1}$ is the annual return and then $\frac{T_{2}}{T_{1}}=12$, or the annual return is twelve times the monthly return. Under the assumption of the distributions being independently distributed variance is additive and hence

$$
\operatorname{VAR}\left(R_{2}\right)=\frac{T_{1}}{T_{2}} \operatorname{VAR}\left(R_{1}\right)
$$

and hence

$$
\sigma_{2}=\sqrt{\frac{T_{2}}{T_{1}} \sigma_{1}}
$$

The regulations proposed in UCITS-for both the $V a R$ limit and the VAR risk-class-do not use an additive definition of returns: percentages are multiplicative. Hence it is wrong to use this square root rule, the estimate of the annual return-scaled from monthly data-will not be a good estimation. 
However in the case of the risk-classes, it reduces only the scale from a ratio scale to an ordinal scale. This is not a big issue, because the risk classes are anyhow intended to reduce VAR (a ratio scale variable) to an ordinal scale. Applying once or twice a non-decreasing scaling does little affect the concept.

In the case of the VaR -limit all risk measures are calculated in the same way and then checked with an arbitrary maximum of $20 \%$ or even relative to each other, so one could assume that applying the square root here will have the same effect. Unfortunately the regulations provide the possibility to calculate $V a R$ on a $95 \%$ base ( $V_{a R_{0.05}}$ in our terminology!) and then scale it back to via this rule and additionally assuming normal distributions it creates a gaping loophole. It allows to select that method that underestimates the tail risk the most (the 5\% VaR calculation, because financial returns tend to be fat-tailed) and then scale it back only as if it was a normal distribution. This is another loophole that masks extreme risks.

\section{REFERENCES}

[1] Artzner P, Delbaen F, Eber JM, Heath D. Thinking coherently. Risk 1997; 68-71.

[2] Bernoulli D. Specimen Theoriae Novae de Mensura Sortis. In Comentarii Academiae Scientiarum Imperialis Petropolitanae 1738; $175-192$.

[3] Fisher I. The nature of capital and income. The Macmillan Company 1906.

[4] Marschak J. Money and the Theory of Assets. Econometrica, Journal of the Econometric Society 1938; 311-325. http://dx.doi.org/10.2307/1905409

[5] Markowitz HM. Portfolio Selection. Journal of Finance. 1952a; 77-91. http://dx.doi.org/10.1111/j.1540-6261.1952.tb01525.x

[6] Rubinstein M. Markowitz's "portfolio selection": A fifty-year retrospective. The Journal of finance 2002; 1041-1045. http://dx.doi.org/10.1111/1540-6261.00453

[7] Markowitz HM. Portfolio Selection: Effcient Diversification of Investments. In John Wiley \& Sons. New York; 1959.

[8] Markowitz HM. Foundations of portfolio theory. Journal of Finance 1991; 469-477.

http://dx.doi.org/10.1111/j.1540-6261.1991.tb02669.x

[9] Markowitz HM. The Utility of Wealth. Journal of Political Economy 1952b; 151-158. http://dx.doi.org/10.1086/257177

[10] Roy AD. Safety First and the Holding of Assets. Econometrica 1952; 431-449. http://dx.doi.org/10.2307/1907413

[11] De Brouwer PJS. Maslowian Portfolio Theory: An alternative formulation of the Behavioural Portfolio Theory. Journal of Asset Management 2009; 359-365.

http://dx.doi.org/10.1057/jam.2008.35

[12] De Brouwer PJS. Target-oriented investment advice. Journal of Asset Management 2011.

[13] Amenc N, Martellini L, Milhau V, Ziemann V. Asset-liability management in private wealth management. The Journal of Portfolio Management 2009; 100-120. http://dx.doi.org/10.3905/JPM.2009.36.1.100

[14] Shefrin H, Statman M. Behavioral Portfolio Theory. Journal of Financial and Quantitative Analysis 2000; 127-151. http://dx.doi.org/10.2307/2676187
[15] Acerbi C, Tasche D. Expected Shortfall: a natural coherent alternative to Value at Risk. Economic Notes 2002a; 379388.

http://dx.doi.org/10.1111/1468-0300.00091

[16] Einstein A. Die Gundlage der allgemeinen Relativitätstheorie. Annalen der Physik 1916; 769-822. http://dx.doi.org/10.1002/andp.19163540702

[17] Acerbi C. Coherent representations of subjective riskaversion. In Risk measures for the 21st century; 2004; 147207.

[18] Pflug GC. Some remarks on the value-at-risk and the conditional value-at-risk. Probabilistic constrained optimization: Methodology and applications 2000; 272-281.

[19] Acerbi C, Tasche D. On the coherence of expected shortfall. In: Szego G. (Ed.), Beyond VaR (Special Issue) of Journal of Banking \& Finance 2002b. http://dx.doi.org/10.1016/s0378-4266(02)00283-2

[20] De Brouwer PJS. Maslowian Portfolio Theory, a Coherent Approach to Strategic Asset Allocation Brussels: VUBPress; 2012.

[21] Acerbi C. Spectral measures of risk: a coherent representation of subjective risk aversion. Journal of Banking \& Finance 2002; 1505-1518.

http://dx.doi.org/10.1016/S0378-4266(02)00281-9

[22] De Brouwer PJS. Comments to CESR/12-1253 and CESR/10-788. on the website of CESR (now ESMA), available via http://www.esma.europa.eu and http://www.debrouwer.com 2010.

[23] De Brouwer PJS. Investment Risk and Risk Classes. unpublished white paper, available on http://www.debrouwer.com 1998.

[24] Artzner P, Delbaen F, Eber JM, Heath D. Coherent measures of risk. Mathematical finance 1999; 203-228. http://dx.doi.org/10.1111/1467-9965.00068

[25] Acerbi C, Simonetti P. Portfolio optimization with spectra measures of risk. preprint 2002.

[26] Black F, Litterman RB. Asset Allocation: Combining investors' views with market equilibrium. The Journal of Fixed Income 1991; 7-18. http://dx.doi.org/10.3905/jif.1991.408013

[27] Black F, Litterman RB. Global portfolio optimization. Financial Analysts Journal 1992; 28-43. http://dx.doi.org/10.2469/faj.v48.n5.28

[28] Central Bank of Ireland. Guidance Note 3/03, Undertakings for Collective Investment in Transferable Securities (UCITS) Financial Derivatives Instruments P.O. Box No 559, Dame Street, Dublin 2, Ireland; 2011.

[29] Dhaene J, Vanduffel S, Goovaerts MJ. Comonotonicity. unpublished, but available via http://en.wikipedia.org/wiki/ Comonotonicity 2007.

[30] Dhaene J, Vanduffel S, Goovaerts MJ, Kaas R, Vyncke D. Comonotonic approximations for optimal portfolio selection problems. Journal of Risk and Insurance 2005; 253-300. http://dx.doi.org/10.1111/j.1539-6975.2005.00123.x

[31] He G, Litterman RB. The intuition behind Black-Litterman model portfolios. Investment Management Research 1999.

[32] Lopez LL. Between Hope and Fear: The Psychology of Risk. Advances in Experimental Social Psychology 1987; 255-295. http://dx.doi.org/10.1016/S0065-2601(08)60416-5

[33] McDonald L, Robinson P. A colossal failure of common sense: The inside story of the collapse of Lehman Brothers: Crown Pub.; 2009.

[34] Rockafellar RT, Uryasev S. Optimization of conditional valueat-risk. Journal of risk 2000; 21-42.

[35] Rockafellar RT, Uryasev S. Conditional value-at-risk for general loss distributions. Journal of Banking \& Finance 2002; 1443-1471.

http://dx.doi.org/10.1016/S0378-4266(02)00271-6 
[36] Satchell S, Scowcroft A. A demystification of the BlackLitterman model: Managing quantitative and traditional portfolio construction. Journal of Asset Management 2000; 138-150.

http://dx.doi.org/10.1057/palgrave.jam.2240011

[37] Strepparava R. Implementation of portfolio optimization with spectral measures of risk; 2009.

[38] Tesler L. Safety First and Hedging. Review of Economic Studies 1955; 1-16.

[39] Tversky A, Kahneman D. The framing of decisions and the psychology of choice. Science 1981; 453-458. http://dx.doi.org/10.1126/science. 7455683

[40] Uryasev S. Conditional value-at-risk: Optimization algorithms and applications. in Computational Intelligence for Financial Engineering, 2000.(CIFEr) Proceedings of the IEEE/IAFE/INFORMS 2000 Conference on 2000; 49-57.

[41] Vanduffel S. Comonotonicity: from risk measurement to risk management Amsterdam: Ph.D. thesis, Universiteit van Amsterdam; 2005.
[42] Vanduffel S, Chen X, Dhaene J, Goovaerts MJ, Henrard L, Kaas R. Optimal approximations for risk measures of sums of lognormals based on conditional expectations. Journal of Computational and Applied Mathematics 2008; 202-218. http://dx.doi.org/10.1016/j.cam.2007.10.050

[43] Vanduffel S, Hoedemakers T, Dhaene J. Comparing approximations for risk measures of sums of nonindependent lognormal random variables. North American Actuarial Journal 2005; 71.

[44] Williams J. The theory of investment value: Fraser; 1938.

[45] Yamai Y, Yoshiba T. Comparative analyses of expected shortfall and value-at-risk under market stress: Bank of Japan; 2002a.

[46] Yamai Y, Yoshiba T. On the Validity of Value-at-Risk: Comparative Analysis with Expected Shortfall. Monetary and economic studies 2002b; 57-86.

Received on 15-02-2016

Accepted on 26-04-2016

Published on 20-07-2016

\section{DOI: http://dx.doi.org/10.6000/2371-1647.2016.02.03}

(C) 2016 Philippe J.S. De Brouwer; Licensee Lifescience Global.

This is an open access article licensed under the terms of the Creative Commons Attribution Non-Commercial License (http://creativecommons.org/licenses/by-nc/3.0/) which permits unrestricted, non-commercial use, distribution and reproduction in any medium, provided the work is properly cited. 